\title{
Concurrent Capecitabine and Radiation Therapy for High-risk Primary Breast Cancer
}

\author{
Uma Goyal $^{1} \bowtie$, Tijana Skrepnik ${ }^{1}$, Rajayogesh Davuluri ${ }^{1}$, Michele B Ley ${ }^{2}$, Pavani Chalasani ${ }^{1}$, Rebecca K \\ Viscusi $^{1}$, Lauren G Lebeau ${ }^{1}$, Robert B Livingston ${ }^{1}$, Justin Famoso ${ }^{1}$, and Victor J Gonzalez ${ }^{1}$ \\ 1. University of Arizona, Tucson, Arizona, United States; \\ 2. Tucson Medical Center, Surgery, Tucson, Arizona, United States
}

$\triangle$ Corresponding author: Uma Goyal, 1501 N. Campbell Ave., PO Box 245081, Tucson, AZ 85724, U.S.A. ugoyal@email.arizona.edu

(c) Ivyspring International Publisher. This is an open access article distributed under the terms of the Creative Commons Attribution (CC BY-NC) license (https://creativecommons.org/licenses/by-nc/4.0/). See http://ivyspring.com/terms for full terms and conditions.

Received: 2016.10.13; Accepted: 2017.01.13; Published: 2017.03.09

\begin{abstract}
Introduction: Data is limited for concurrent capecitabine-radiotherapy (CCRT) in primary breast cancer. We evaluated outcomes and toxicities of patients at high risk of locoregional recurrence receiving adjuvant CCRT.

Methods: Ten non-metastatic breast cancer patients receiving concurrent treatment were reviewed retrospectively. Capecitabine was given during and after radiation. Toxicity was reviewed using weekly on-treatment visit and follow-up notes.

Results: All patients had stage II-III disease. Four patients had grade 3 skin toxicity during radiation. Capecitabine and RT-related toxicity breaks occurred for 5 and 0 patients, respectively. At 1-month follow-up, 9 patients recovered from acute toxicities sufficiently to start adjuvant capecitabine. At 25 months median follow-up, 1 patient had synchronous local recurrence and distant metastasis (DM), while 3 patients had DM only.

Conclusions: Use of CCRT for breast cancer was associated with significant acute grade 3 dermatitis, however, all patients successfully completed their radiation course without interruption.
\end{abstract}

Key words: breast cancer, capecitabine, high-risk, radiation

\section{Introduction}

High-risk breast cancer (HRBC), defined as bulky, invasive disease with skin, chest wall and nodal involvement, is composed mainly of stage II-III diagnoses. Five-year survivals are approximately $70 \%-48 \%$ for stage IIB-IIIB disease [1,2].

Standard of care for HRBC includes neoadjuvant chemotherapy (NAC) followed by surgery and adjuvant radiotherapy (RT) $[3,4,5]$. Adjuvant RT is associated with improved loco-regional control (LRC), breast-cancer specific mortality, disease-free survival (DFS), and overall survival (OS) [6-11].

Capecitabine has been used with increasing frequency for the treatment of metastatic breast cancer
$[12,13]$. Multiple investigators have reported on the use of concurrent capecitabine-RT (CCRT) for metastatic or recurrent breast cancer [14-18]. Factors predictive of response to capecitabine with or without paclitaxel, include single-organ metastasis, ER-positivity and Her-2-negativity [19-22]. A meta-analysis has shown an overall response with capecitabine-based chemotherapy versus capecitabine-free chemotherapy. OS was found to be non-significantly higher for capecitabine-based chemotherapy [12]. In randomized studies of non-metastatic patients undergoing NAC regimens, capecitabine was well-tolerated with mixed results 
regarding DFS $[23,24]$.

To our knowledge, there are no reports of the tolerability, toxicity and efficacy of single-agent capecitabine with RT for primary HRBC. We evaluated primary HRBC patients treated with NAC followed by CCRT after surgery. Acute and late toxicity, local recurrence (LR), distant metastases $(\mathrm{DM})$, and OS are reported at a median follow-up of 25 months.

\section{Materials and Methods}

Records of HRBC patients treated between 2009-2014 were evaluated retrospectively after study approval by the institutional review board. Inclusion criteria were non-metastatic, locally-advanced and biopsy-proven HRBC receiving CCRT, with cT2-4N0-3 or pT1c-2N0-3 based on the American Joint Committee on Cancer version7. High-risk disease was defined based on minimal response to neoadjuvant therapy in patients with stage II-III disease.

We identified ten patients treated with CCRT. All patients received NAC or neoadjuvant endocrine therapy. All patients were deemed operable by the treating surgeon. The use of CCRT was at the discretion of the treating medical and radiation oncologists. Indications included persistently elevated ki-67 levels at the time of surgery $(>50 \%)$, significant axillary tumor burden $(>50 \%$ dissected lymph nodes involved) and persistent disease at the primary site.

\section{Chemotherapy}

Capecitabine was $1000 \mathrm{mg}$ p.o. twice a day (b.i.d.) for 2 cycles during RT and 4 cycles of $1000-1500 \mathrm{mg}$ p.o. b.i.d. after RT completed. A cycle was defined as 2 weeks on treatment then 1 week off. Capecitabine was discontinued at the discretion of the treating radiation oncologist based on toxicity during CCRT.

\section{Radiation Therapy}

RT was 45-50.4Gy in 1.8-2Gy daily fractions to the chest wall or breast and regional lymph nodes followed by a 10-14Gy boost to the scar or lumpectomy cavity. 3D-conformal RT was delivered with tangential fields and a supraclavicular field, with inclusion of internal mammary nodes in 2 cases using wide tangent fields.

\section{Endpoints}

Acute toxicity was defined as occurring at treatment start to 3 months after completion of treatment. Late radiation effects occurred $>3$ months after completion of treatment. End-points evaluated were acute and late toxicity grade per Common Terminology Criteria for Adverse Events version 4.0, local recurrence free survival (LRFS), distant recurrence free survival (DRFS), and OS. Distant disease was defined as disease found beyond the treated breast and axilla on follow-up imaging or clinical exam.

\section{Follow-up}

All patients were evaluated weekly during CCRT, 1-month after completion of RT, and routinely thereafter. Toxicity was assessed by review of weekly on-treatment (OTV) and follow-up notes.

\section{Statistical Methods}

The Fisher exact test was used to assess the association between toxicity at the last treatment and toxicity at 1-month and 6-month follow-up. Kaplan-Meier curves were used to calculate the LRFS and DRFS. The log-rank test was used to compare LRFS and DRFS between patients who received capecitabine as planned and those in whom capecitabine was held. OS for the cohort was calculated and plotted using Kaplan-Meier curves and death was counted if reported within the electronic medical record.

\section{Results}

All patients received neoadjuvant systemic therapy followed by surgery (cytotoxic chemotherapy=9). One patient received neoadjuvant hormonal therapy alone due to a low OncotypeDX score from the biopsy specimen.

Seven patients underwent modified radical mastectomy. One patient had two different primary tumors in the same breast at the time of diagnosis, Luminal B histology and the other Basal histology. Biologic subtype was Luminal $B \quad n=9$ on immunohistochemistry. Eight patients had residual breast involvement with pT1-2 disease. Seven patients had residual nodal involvement at the time of surgery.

Seven patients with Luminal B disease received adjuvant hormonal therapy. Two patients did not receive adjuvant hormonal therapy because they were lost to follow-up. None of the patients received adjuvant chemotherapy. Patient and lesion characteristics are provided in Table $1 \mathrm{~A}$ and $\mathrm{B}$, respectively.

\section{Toxicity during treatment}

Three patients developed grade 1 radiation dermatitis, 3 grade 2, 4 grade 3 , and none with grade 4. Capecitabine was held for 5 patients due to skin toxicity, of whom 2 patients developed brisk confluent erythema. Four RT plans required 
modification due to grade 3 skin toxicity; the boost was started early at 44Gy for one patient, the bolus was discontinued for 2 patients, and the internal mammary field was discontinued at 46Gy (planned $50 \mathrm{~Gy}$ ) for 1 patient. No patient required a break from RT.

\section{Toxicity at follow-up}

We evaluated skin toxicity at 1- and 6-month follow-up to see if the effects were acute vs. late. At the 1-month follow-up, 3 patients who had grade 3 toxicity during RT had grade 1 toxicity, with 1 patient having no toxicity. None of the patients who had <grade 3 skin toxicity at the end of treatment had skin toxicity at 1-month follow-up. There were no grade 4 toxicities documented at 1-month follow-up. Nine patients had recovered from their acute toxicities sufficiently to start adjuvant capecitabine by the 1-month follow-up. Patients with $\geq$ grade 3 skin toxicity at the end of treatment were more likely to have skin toxicity at 1-month follow-up compared to patients with < grade 3 toxicity ( $\mathrm{p}=0.03$, Table 2 ).

Follow-up at 6-months was available for 7 patients. One patient had no reported toxicity, 3 had grade 1 chest wall pain, and 3 had grade 1 lymphedema. Three patients who had grade 3 skin toxicity at the end of treatment had follow-up data at 6-months. Two patients had grade 1 lymphedema and 1 had grade 1 chest wall pain. Four patients had <grade 3 skin toxicity at the end of treatment had 6-month follow-up. Of these patients, 1 had no toxicity, 2 had grade 1 chest wall pain, and 1 had grade 1 lymphedema. Patients with $\geq$ grade 3 skin toxicity at the end of treatment were not more likely to have skin toxicity at the 6-month follow-up compared to patients with <grade 3 toxicity ( $\mathrm{p}=1.0$, Table 2$)$.

At a median follow-up of 25 months, there were no documented cases of pneumonitis or brachial plexopathy. Table 3 includes the major toxicities seen at last OTV, 1, 3, and 6-month follow-up.

\section{Disease outcomes}

At a median follow-up of 25 months, one patient had synchronous LR and DM while three patients had DM only. No patients had isolated LR. The 2-year LRFS for the entire cohort was $87 \%$ (Figure 1a), and the DRFS was 75\% (Figure 2a). The disease outcomes of patients for whom capecitabine was held during RT vs not held showed no difference in either LRFS $(p=0.32)$ or DRFS $(p=0.76)$ (Figures $1 b$ and $2 b$, respectively). All patients are currently alive with 2-year OS 100\%.
Table 1. Patient and Lesion Characteristics

\begin{tabular}{|c|c|c|}
\hline & Characteristic & n(\%) \\
\hline \multicolumn{3}{|l|}{ A. } \\
\hline \multicolumn{3}{|l|}{ Patients $(n=10)$} \\
\hline \multirow[t]{6}{*}{ Age(years) } & $30-39$ & $3(30)$ \\
\hline & $40-49$ & $2(20)$ \\
\hline & $50-59$ & $1(10)$ \\
\hline & $60-69$ & $3(30)$ \\
\hline & $70-79$ & $0(0)$ \\
\hline & $80-89$ & $1(10)$ \\
\hline \multirow[t]{4}{*}{ BMI } & $<25$ & $5(50)$ \\
\hline & $25-29.9$ & $3(30)$ \\
\hline & $30-39.9$ & $1(10)$ \\
\hline & Unknown & $1(10)$ \\
\hline \multirow[t]{3}{*}{ Neoadjuvant treatment } & Chemotherapy & $9(90)$ \\
\hline & Hormone therapy & $1(10)$ \\
\hline & Receptor antibody* & $1(10)$ \\
\hline \multirow[t]{3}{*}{ Adjuvant treatment } & Chemotherapy & $0(0)$ \\
\hline & Hormone therapy & $7(70)$ \\
\hline & Receptor antibody & $0(0)$ \\
\hline \multirow[t]{2}{*}{ Surgery type } & Breast-conserving & $3(30)$ \\
\hline & Mastectomy & $7(70)$ \\
\hline \multicolumn{3}{|l|}{$\begin{array}{l}\text { B. } \\
\text { Lesions }(n=11)\end{array}$} \\
\hline \multirow[t]{4}{*}{ Subtype } & LuminalA & $0(0)$ \\
\hline & LuminalB & $9(82)$ \\
\hline & Her-2-neu positive & $0(0)$ \\
\hline & Basal & $2(18)$ \\
\hline \multirow[t]{3}{*}{ Grade } & 1 & $1(9)$ \\
\hline & 2 & $0(0)$ \\
\hline & 3 & $10(91)$ \\
\hline \multirow[t]{3}{*}{ Lymphovascular invasion } & Present & $5(45)$ \\
\hline & Absent & $3(27)$ \\
\hline & Not reported & $3(27)$ \\
\hline \multirow[t]{4}{*}{ Clinical T Stage } & 1 & $0(0)$ \\
\hline & 2 & $6(55)$ \\
\hline & 3 & $3(27)$ \\
\hline & 4 & $2(18)$ \\
\hline \multirow[t]{4}{*}{ Clinical N Stage } & 0 & $2(18)$ \\
\hline & 1 & $4(36)$ \\
\hline & 2 & $3(27)$ \\
\hline & 3 & $2(18)$ \\
\hline \multirow[t]{6}{*}{ Pathologic T Stage } & In-situ & $1(9)$ \\
\hline & 0 & $2(18)$ \\
\hline & 1 & $5(45)$ \\
\hline & 2 & $3(27)$ \\
\hline & 3 & $0(0)$ \\
\hline & 4 & $0(0)$ \\
\hline \multirow[t]{4}{*}{ Pathologic N Stage } & 0 & $4(36)$ \\
\hline & 1 & $2(18)$ \\
\hline & 2 & $3(27)$ \\
\hline & 3 & $2(18)$ \\
\hline
\end{tabular}

${ }^{*} 1$ patient received receptor antibody with chemotherapy on a clinical trial 
Table 2. The Statistical Significance of Toxicity Post-treatment

\begin{tabular}{lllll}
\hline \multicolumn{3}{c}{$\begin{array}{l}\text { Toxicity at 1month after } \\
\text { treatment }\end{array}$} & $\begin{array}{l}\text { Toxicity at 6months after } \\
\text { treatment }\end{array}$ \\
\hline & Yes & No & Yes & No \\
\hline$\geq$ Grade3 & $75.00 \%(3 / 4)$ & $25 \%(1 / 4)$ & $100.00 \%(3 / 3)$ & $0.00 \%(0 / 3)$ \\
<Grade3 & $0 \%(0 / 6)$ & $100 \%(6 / 6)$ & $75.00 \%(3 / 4)$ & $25.00 \%(1 / 4)$ \\
p-value & 0.03 & & 1.0 & \\
\hline
\end{tabular}

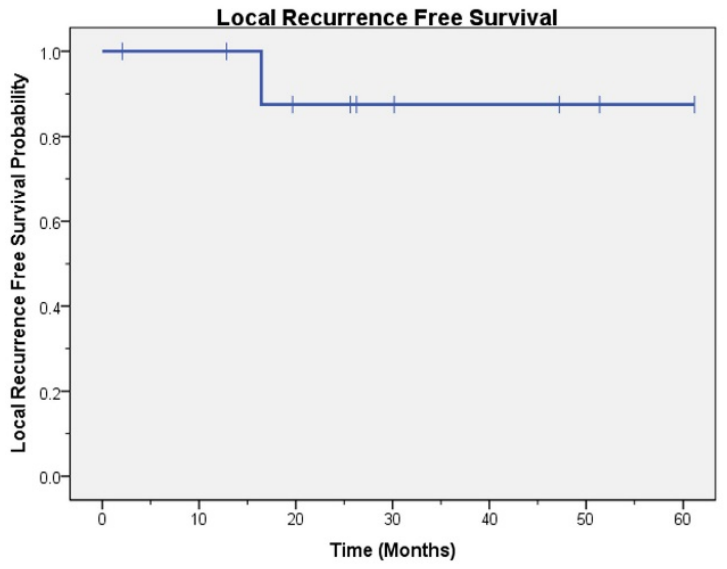

(A)
Table 3. Number of Patients with Specific Toxicity

\begin{tabular}{|c|c|c|c|c|c|c|c|c|}
\hline & \multicolumn{2}{|c|}{ Last OTV } & \multicolumn{2}{|c|}{ 1month } & \multicolumn{2}{|c|}{ 3month } & \multicolumn{2}{|c|}{6 month } \\
\hline & $\begin{array}{l}\text { Grad } \\
\text { e } 1-2\end{array}$ & $\begin{array}{l}\text { Grad } \\
\text { e 3-4 }\end{array}$ & $\begin{array}{l}\text { Grad } \\
\text { e } 1-2\end{array}$ & $\begin{array}{l}\text { Grad } \\
\text { e 3-4 }\end{array}$ & $\begin{array}{l}\text { Grad } \\
\text { e } 1-2\end{array}$ & $\begin{array}{l}\text { Grad } \\
\text { e 3-4 }\end{array}$ & $\begin{array}{l}\text { Grad } \\
\text { e } 1-2\end{array}$ & $\begin{array}{l}\text { Grad } \\
\text { e 3-4 }\end{array}$ \\
\hline $\begin{array}{l}\text { Hyperpigmentat } \\
\text { ion }\end{array}$ & 0 & 0 & $2 / 10$ & $0 / 10$ & $2 / 9$ & $0 / 9$ & $0 / 7$ & 0 \\
\hline Lymphedema & 0 & 0 & $2 / 10$ & $0 / 10$ & $2 / 9$ & $0 / 9$ & $3 / 7$ & 0 \\
\hline $\begin{array}{l}\text { Radiation } \\
\text { dermatitis }\end{array}$ & $6 / 10$ & $4 / 10$ & $1 / 10$ & $0 / 10$ & $1 / 9$ & $0 / 9$ & $0 / 7$ & 0 \\
\hline Chest wall pain & 0 & 0 & $0 / 10$ & $0 / 10$ & $0 / 9$ & $0 / 9$ & $2 / 7$ & 0 \\
\hline $\begin{array}{l}\text { Soft tissue } \\
\text { fibrosis }\end{array}$ & 0 & 0 & 0 & 0 & $2 / 9$ & 0 & 0 & 0 \\
\hline
\end{tabular}

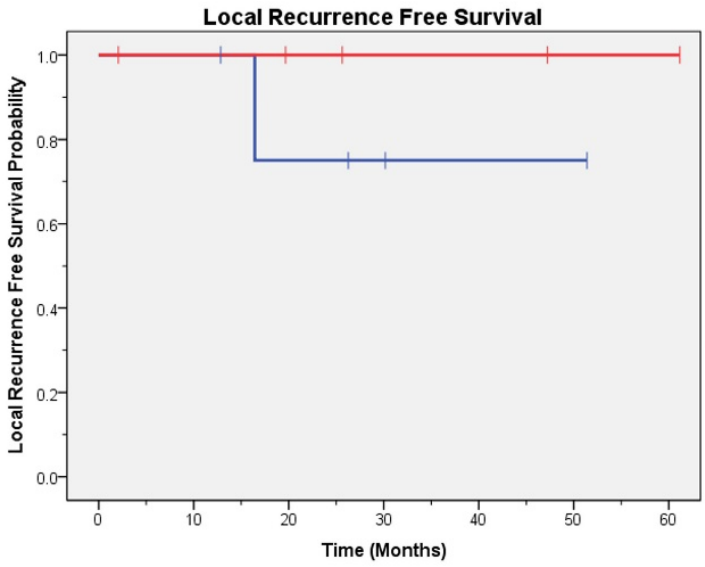

(B)

Figure 1. Local Recurrence Free Survival. The Kaplan-Meier local recurrence free survival curve (A) for all patients; (B) by capecitabine treatment are provided (blue line capecitabine not held and red line capecitabine held).

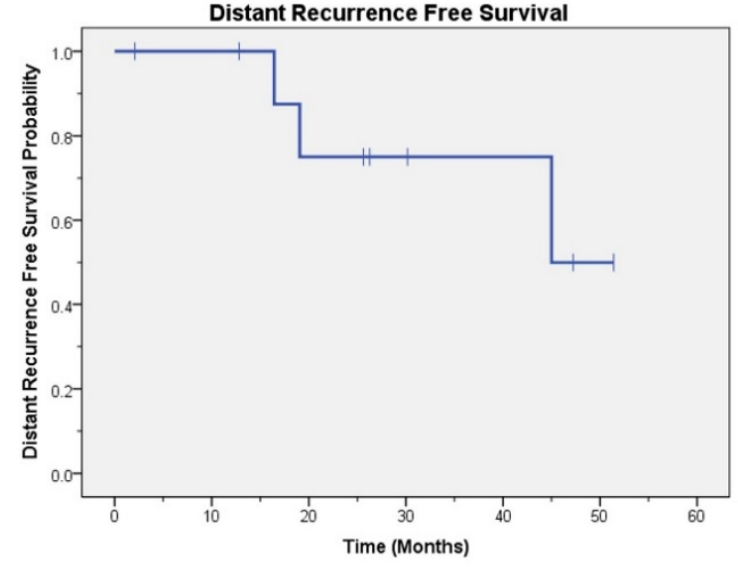

(A)

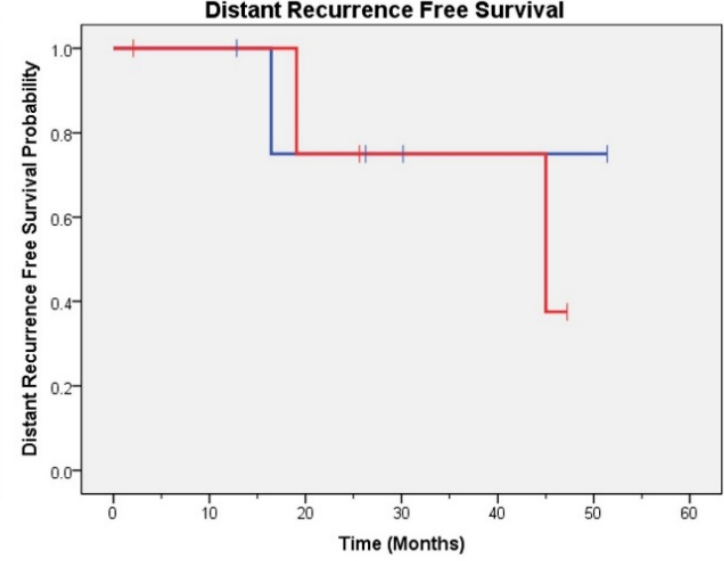

(B)

Figure 2. Distant Recurrence Free Survival. The Kaplan-Meier distant recurrence free survival curve (A) for all patients; (B) by capecitabine treatment are provided (blue line capecitabine not held and red line capecitabine held).

\section{Discussion}

Patients with residual disease following neoadjuvant chemotherapy are at significant risk of locoregional recurrence [25]. Therefore, this population may warrant intensification of adjuvant therapy. In this study, we analyzed outcomes in patients with HRBC who received adjuvant CCRT. Seven patients had residual nodal disease at the time of surgery after neoadjuvant systemic therapy. 
Distant recurrence was the predominant mode of failure in our series with one patient failing locally and distantly and 3 patients failing distantly only.

In our study, 9 patients had recovered from their acute skin toxicity at the 1-month follow-up to start adjuvant capecitabine. A current phase II study at MD Anderson Cancer Center assessing neoadjuvant capecitabine and RT in advanced breast cancer is anticipated for primary completion in 2017 and should help assess the utility and potential toxicities of this regimen.

Capecitabine has demonstrated activity in metastatic and inoperable breast cancer and has been used as a radiosensitizer with concurrent RT in other disease sites [26]. We had higher than expected acute dermatitis with adjuvant CCRT but limited late toxicity. Given our low numbers and limited long-term follow-up, we must be careful to limit generalizations regarding late toxicity. There were, however, no unanticipated late toxicities noted; this includes one patient who underwent tissue expander placement followed by an implant reconstruction 2 years after completion of RT and another patient who had pre-existing implants at the time of RT who had no evidence of capsular contracture at 2-year follow-up.

\section{Conclusions}

CCRT appears to be associated with increased acute dermatitis but acceptable late toxicity. Further prospective studies are warranted to elucidate whether adding chemotherapy to adjuvant RT in HRBC will result in improved outcomes.

\section{Competing Interests}

The authors have declared that no competing interest exists.

\section{References}

1. American Cancer Society. American Cancer Society Breast Cancer Facts and Figures 2007-2008. Atlanta, GA: American Cancer Society, Inc. 2009.

2. [Internet] SEER. http://seer.cancer.gov/statfacts/html/breast.html.

3. Cortazar P, Zhang L, Untch M, et al. Pathological complete response and long-term clinical benefit in breast cancer: the CTNeoBC pooled analysis. Lancet. 2014;384(9938):164-172.

4. Carey LA, Winer EP. Defining success in neoadjuvant breast cancer trials. Lancet. 2014;384(9938):115-116.

5. Mieog JS, van der Hage JA, van de Velde CJ. Preoperative chemotherapy for women with operable breast cancer. Cochrane Database Syst Rev. 2007

6. Buchholz TA, Haffty BG. Breast Cancer: Locally advanced and recurrent disease, postmastectomy radiation, and systemic therapies. In: Halperin EC, Perez CA, Brady LW, eds. Principles and Practice of Radiation Oncology,5th edition. New York, NY: Lippincott Williams and Wilkins; 2008:1291-1317.

7. Recht A, Edge SB, Solin LJ, et al. Postmastectomy radiotherapy: clinical practice guidelines of the American Society of Clinical Oncology. J Clin Oncol. 2001;19:1539-1569.

8. Taylor ME, Haffty BG, Rabinovitch R, et al. ACR appropriateness criteria on postmastectomy radiotherapy expert panel on radiation oncology-breast. Int J Radiat Oncol Biol Phys. 2009;73:997-1002.
9. Ragaz J, Jackson SM, Le N, et al. Adjuvant radiotherapy and chemotherapy in node-positive premenopausal women with breast cancer. N Engl J Med. 1997;337:956-962.

10. Overgaard M, Hansen PS, Overgaard J, et al. Postoperative radiotherapy in high-risk premenopausal women with breast cancer who receive adjuvant chemotherapy. Danish Breast Cancer Cooperative Group 82b Trial. N Engl J Med. 1997;337:949-955.

11. Overgaard M, Jensen MB, Overgaard J, et al. Postoperative radiotherapy in high-risk post-menopausal breast cancer patients given adjuvant tamoxifen: Danish Breast Cancer Cooperative Group DBCG 82c randomized trial. Lancet. 1999;353:1641-1648.

12. Yin W, Pei G, Liu G, et al. Efficacy and safety of capecitabine-based first-line chemotherapy in advanced or metastatic breast cancer: a meta-analysis of randomised controlled trials. Oncotarget. 2015;6(36):39365-39372.

13. Shankar A, Roy S, Rath GK, et al. Aromatase Inhibition and Capecitabine Combination as 1st or 2nd Line Treatment for Metastatic Breast Cancer-a Retrospective Analysis. Asian Pac J Cancer Prev. 2015;16(15):6359-6364.

14. Shaughnessy JN, Meena RA, Dunlap NE, et al. Efficacy of Concurrent Chemoradiotherapy for patients with locally recurrent or Advanced Inoperable Breast Cancer. Clinical Breast Cancer. 2015;15(2):135-142.

15. Oshaughnessy JA, Blum J, Moiseyenko V, et al. Randomized, open-label, phase II trial of oral capecitabine (Xeloda) vs. a reference arm of intravenous CMF (cyclophosphamide, methotrexate and 5-fluorouracil) as first-line therapy for advanced/metastatic breast cancer. Ann Oncol. 2001;12(9):1247-1254.

16. Fumoleau P, Largillier R, Clippe C, et al. Multicentre, phase II study evaluating capecitabine monotherapy in patients with anthracycline-and taxane-pretreated metastatic breast cancer. Eur J Cancer. 2004;40(4):536-542.

17. Yamamoto D, Iwase S, Tsubota $Y$, et al. Randomized study of orally administered fluorinated pyrimidines (capecitabine versus S-1) in women with metastatic or recurrent breast cancer: Japan Breast Cancer Research Network 05 Trial. Cancer Chemother Pharmacol. 2015;75(6):1183-1189.

18. Wurschmidt F, Dahle J, Petersen C, et al. Reirradiation of recurrent breast cancer with and without concurrent chemotherapy. Radiat Oncol. 2008;3:28.

19. Hong JY, Park YH, Choi MD, et al. Characterization of Durable Responder for Capecitabine Monotherapy in Patients with Anthracycline and Taxane-pretreated metastatic breast cancer. Clin Breast Cancer. 2015;15(5):e287-92.

20. Babacan T, Efe O, Hasirci AS, et al. Efficacy of capecitabine monotherapy as the first-line treatment of metastatic HER-2 negative breast cancer. Tumori. 2015;101(4):418-423.

21. Levin MK, Wang $K$, Yelensky $R$, et al. Genomic alterations in DNA repair and chromatin remodeling genes in estrogen receptor-positive metastatic breast cancer patients with exceptional responses to capecitabine. Cancer Med. 2015;4(8):1289-1293.

22. Kaufman PA, Awada A, Twelves C, et al. Phase III open-label randomized study of eribulin mesylate versus capecitabine in patients with locally advanced or metastatic breast cancer previously treated with an anthracycline and a taxane. J Clinical Oncol. 2015;33(6):594-601.

23. Eremin J, Cowley G, Walker LG, et al. Women with large $(>/=3 \mathrm{~cm})$ and locally advanced breast cancers (T3,4,N1,2,M0) receiving neoadjuvant chemotherapy (NAC: cyclophosphamide, doxorubicin, docetaxel): addition of capecitabine improves 4-year disease free survival. Springerplus. 2015;4:9.

24. Yoo C, Kim SB, Ahn JH, et al. A Randomized Phase II Trial of Capecitabine plus Vinorelbine followed by Docetaxel versus Adriamycin plus Cyclophosphamide followed by Docetaxel as neoadjuvant chemotherapy for breast cancer. Cancer Res Treat. 2014;47(3):406-415.

25. Mamounas EP, Anderson SJ, Dignam JJ, et al. Predictors of Locoregional recurrence after neoadjuvant chemotherapy: Results from combined analysis of national surgical adjuvant breast and bowel project B-18 and B-27. J Clin Oncol. 2012;30(32):3960-3966.

26. Velenik V, Anderluh F, Oblak I, et al. Capecitabine as a radiosensitizing agent in neoadjuvant treatment of locally advanced resectable rectal cancer:prospective phase II trial. Croat Med J. 2006;47(5):693-700. 